\title{
High-Potency Topical Steroid Treatment of Multiple Keratoacanthomas Associated With Prurigo Nodularis
}

Malia B. Downing, MD; Megan Prouty, MD; Dominic J. Wu, MD; Anand Rajpara, MD

Patients who present with multiple keratoacanthomas (KAs) associated with prurigo nodularis often pose a treatment challenge. These lesions often require aggressive treatment, such as Mohs micrographic surgery, surgical excision, electrodesiccation and curettage, intralesional steroid injection, and long-term acitretin. 5-Fluorouracil (5-FU) cream 5\% has been shown to be effective; however, topical options are limited when 5-FU fails. We have found success using a high-potency topical steroid under occlusion, resulting in resolution of $\mathrm{KAs}$ and prurigo nodules.

Cutis. 2021;107:221-222.

\section{Practice Gap}

Multiple keratoacanthomas (KAs) of the legs often are a challenge to treat, especially when these lesions appear within a field of prurigo nodules. Multiple KAs associated with prurigo nodularis is a rarer finding; more often, the condition is reported on the lower limbs of elderly women with actinically damaged skin. ${ }^{1,2}$ At times, it can be difficult to distinguish between KA and prurigo nodularis in these patients, who often report notable pruritus and might have associated eczematous dermatitis. ${ }^{2}$

Keratoacanthomas often are treated with aggressive modalities, such as Mohs micrographic surgery, excision, and electrodesiccation and curettage. Some patients are hesitant to undergo surgical treatment, however, preferring a less invasive approach. Trauma from these aggressive modalities also can be associated with recurrence of existing lesions or development of new KAs, possibly related to stimulation of a local inflammatory response and upregulation of helper T cells..$^{-4}$

Acitretin and other systemic retinoids often are considered first-line therapy for multiple KAs. Cyclosporine has been added as adjunctive treatment in cases associated with prurigo nodularis or eczematous dermatitis ${ }^{1,2}$; however, these treatments have a high rate of discontinuation because of adverse effects, including transaminitis, xerostomia, alopecia (acitretin), and renal toxicity (cyclosporine). ${ }^{2}$

Another treatment option for patients with coexisting KA and prurigo nodularis is intralesional corticosteroids, often administered in combination with systemic retinoids. ${ }^{3}$ Topical 5 -fluorouracil $(5-\mathrm{FU})$ has been used successfully for KA, but topical treatment options are limited if 5-FU fails. Topical imiquimod and cryotherapy are thought to be of little benefit, and the appearance of new KA within imiquimod and cryotherapy treatment fields has been reported. ${ }^{1,2}$ Topical corticosteroids have been used as an adjuvant therapy for multiple KAs associated with prurigo nodularis; however, a PubMed search of articles indexed for MEDLINE using the terms keratoacanthoma and steroid and keratoacanthoma and prurigo nodularis yielded no published reports of successful use of topical corticosteroids as monotherapy. ${ }^{2}$

From the Department of Dermatology, University of Kansas Medical Center, Kansas City.

The authors report no conflict of interest.

Correspondence: Malia B. Downing, MD, Department of Dermatology, University of Kansas Medical Center, MS 2025, Kansas City, KS 66160 (mdowning3@kumc.edu).

doi: $10.12788 /$ cutis. 0221 
A, Multiple keratoacanthomas on the shin prior to treatment. B, Resolution of keratoacanthomas after treatment with a highpotency topical steroid under occlusion.
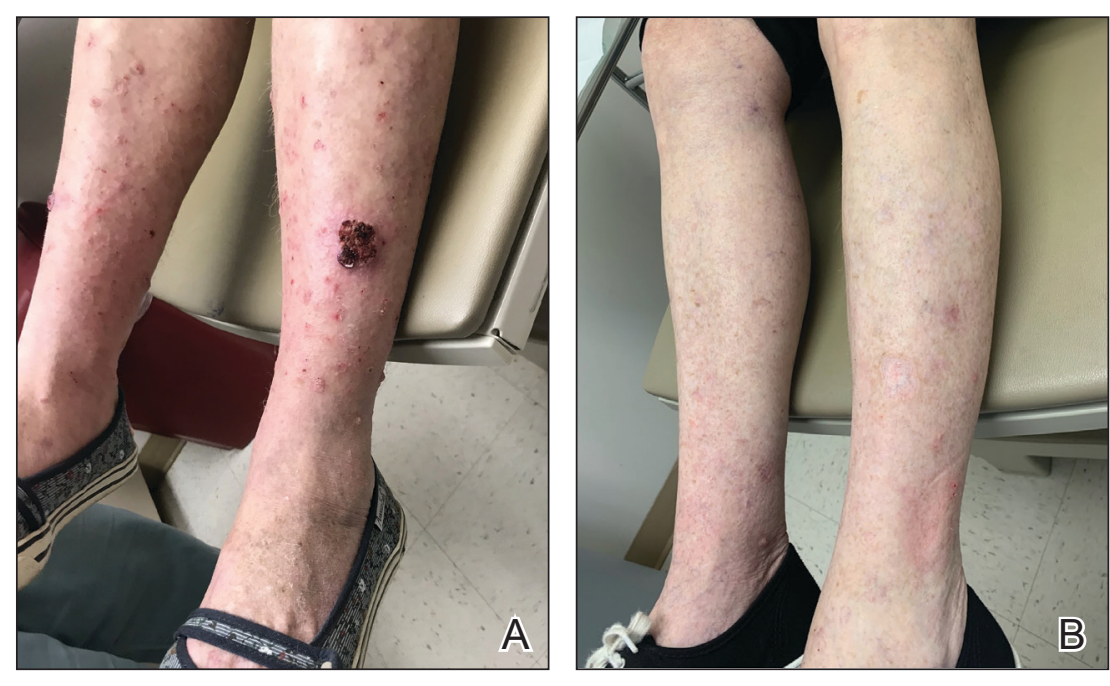

\section{The Technique}

For patients who want to continue topical treatment of coexisting KA and prurigo nodularis after topical 5-FU fails, we have found success applying a high-potency topical corticosteroid to affected areas under occlusion nightly for 6 to 8 weeks. This treatment not only leads to resolution of $\mathrm{KA}$ but also simultaneously treats prurigo nodules that might be clinically difficult to distinguish from KA in some presentations. This regimen has been implemented in our practice with remarkable reduction of KA burden and relief of pruritus.

In a 68-year-old woman who was treated with this technique, multiple biopsies had shown KA (or welldifferentiated squamous cell carcinoma that appeared clinically as KA) on the shin (Figure, A) arising amid many lesions consistent with prurigo nodules. Topical 5-FU had failed, but the patient did not want to be treated with a more invasive modality, such as excision or injection.

Instead, we treated the patient with clobetasol propionate ointment $0.05 \%$ under occlusion nightly for 6 weeks. This strategy produced resolution of both KA and prurigo nodules (Figure, B). When lesions recurred after a few months, they were successfully re-treated with topical clobetasol under occlusion in a second 6-week course.

\section{Practical Implications}

Treatment of multiple KAs associated with prurigo nodularis can present a distinct challenge. For the subset of patients who want to pursue topical treatment, options reported in the literature are limited. We have found success treating multiple KAs and associated prurigo nodules with a high-potency topical corticosteroid under occlusion, with minimal or no adverse effects. We believe that a topical corticosteroid can be implemented easily in clinical practice before a more invasive surgical or intralesional modality is considered.

\section{REFERENCES}

1. Kwiek B, Schwartz RA. Keratoacanthoma (KA): an update and review. J Am Acad Dermatol. 2016;74:1220-1233. doi:10.1016/j. jaad.2015.11.033

2. Wu TP, Miller K, Cohen DE, et al. Keratoacanthomas arising in association with prurigo nodules in pruritic, actinically damaged skin. J Am Acad Dermatol. 2013;69:426-430. doi:10.1016/J.JAAD.2013.03.035

3. Sanders S, Busam KJ, Halpern AC, et al. Intralesional corticosteroid treatment of multiple eruptive keratoacanthomas: case report and review of a controversial therapy. Dermatol Surg. 2002;28:954-958. doi:10.1046/j.1524-4725.2002.02069.x

4. Lee S, Coutts I, Ryan A, et al. Keratoacanthoma formation after skin grafting: a brief report and pathophysiological hypothesis. Australas J Dermatol. 2017;58:E117-E119. doi:10.1111/ajd.12501 